\title{
Brain arrest, cardiac arrest and uncertainties in defining death
}

\author{
Sam D. Shemie*
}

$\mathrm{I}_{\mathrm{n}}$ brain death in Latin America, as evaluated retrospectively in seven Brazilian pediatric intensive care units (PICU), and demonstrate some revealing observations. The investigators document substantial geographic variation in the diagnosis suggestive of differences in applying the clinical examination, rather than differences in case mix. Similar to the variabilities in diagnostic criteria described in other countries, ${ }^{2,3}$ there is also lack of uniformity in the use of complementary exams. In northeastern and southeastern Brazil, the extended time interval from diagnosis of death to the removal of support technology is of particular concern. This indicates that many physicians are concerned about stopping 'life' support technology after brain 'death' is diagnosed. The extremely poor incidence of organ donation reflects a discomfort with the issues of death and the use of human organs for transplantation in Brazilian society, and within the Brazilian health care profession.

Despite the widespread international acceptance of brain death criteria, these troubling findings are not isolated to Brazil. Medicine and society continue to struggle with the definition of death, especially in view of advances in complex life support systems. Our ability to support organ failure with technology and transplantation raises important questions of when a disease is irreversible, when further treatment is no longer effective or when death has occurred. The life preserv-

* MD. Division of Pediatric Critical Care. Medical Director, Extracorporeal Life Support Program, Montreal Children's Hospital, McGill University Health Centre, Montreal, Canada. The Bertram Loeb Chair in Organ and Tissue Donation, Faculty of Arts, University of Ottawa, Ottawa, Canada. Chair, Donation Committee, Canadian Council for Donation and Transplantation.

Suggested citation: Shemie SD. Brain arrest, cardiac arrest and uncertainties in defining death. J Pediatr (Rio J). 2007;83(2):102-104.

doi:10.2223/JPED. 1607 ing benefits of organ donation, as an option arising after death is established, demands clear definitions. The study by Lago et al. ${ }^{1}$ cannot answer whether these reluctant practices reflect the uncertain beliefs and perceptions of Brazilian practitioners, or simply a matter of insufficient professional education.

Brain death is a term and a concept that remains a continuing source of misunderstanding. For practitioners and families, it may be difficult to comprehend 'death' in an individual whose vital signs - heartbeat, the warmth of circulation and tidal movement of the lungs - are maintained by support technology. The concept of brain death itself has been criticized as a social construct, created for utilitarian purposes to permit transplantation. ${ }^{4}$ All these factors lead to a lingering perception, as suggested by Lago's study, that brain death is, perhaps, a false concept. If brain death is not really death at all, then stopping life support is not mandated and organ donation is not relevant.

Well, what does it mean to be 'dead'? Is death an event, process or a transition? Medical literature does not clearly distinguish the different ways that 'death' may be defined eg. medical, legal, religious, spiritual, existential, philosophical, supernatural, mystical. ICU practitioners remain confused. ${ }^{5}$ However, in medicine and law, the separation between being alive and dead is quite clear. Death is the point in time when concrete consequences occur, including no legal requirement to provide resuscitation or life support technologies, loss of personhood and individual rights, potential for organ donation and autopsy, execution of legal will and estate, life insurance and disposition of the body by burial or cremation.

Organ support and replacement technologies also teach us about the mechanics of death. There are three basic physiological mechanisms: a) primary cardiac arrest leading to arrest of the circulation b) primary respiratory arrest, which via hypoxemia causes a secondary cardiac arrest, or c) primary brain arrest, which via interruption of airway control and res- 
piratory drive, causes a secondary respiratory then cardiac arrest. Regardless of initial disease state, all critical illnesses threaten life in this way. Interruption of this sequence with various forms of life support is fundamental to ICU practice. Life-sustaining technologies are started, with the use of artificial airways, mechanical ventilation, hemodynamic support with inotropes-vasopressors and renal replacement therapies. Advanced support may include extracorporeal systems such as extracorporeal membrane oxygenation (ECMO) and artificial hearts (ventricular assist devices such as the Berlin heart). The principle behind their application is to sustain vital function, to allow time or treatment (medical or surgical) to reverse the underlying life-threatening state.

Although access to technology varies from country to country, medicine has advanced to the point that all vital organs (heart, lung, liver, kidney) can be supported by machines, or replaced by transplantation. Complete and irreversible arrest of the heart is not death, as long as oxygenated circulation to the body can be provided mechanically, with the use of extracorporeal support such heart-lung bypass machines or ECMO. Circulation can be artificially maintained and the arrested heart can then be replaced by transplantation. The event may be the cardiac arrest, but death only occurs if it leads to an accompanying loss of circulation.

The brain is the only organ that cannot be supported by technology or replaced by transplantation. For all forms of severe brain injury, ICU care does not replace any functions of the brain. Mechanical ventilation merely interrupts the way brain failure leads to death and neuroprotective therapies limit secondary brain injury. It was once thought that brain death consistently leads to cardiac arrest because of the associated hemodynamic instability. ${ }^{6}$ It is now well known that any degree of brain failure, including brain death, can be sustained indefinitely with mechanical ventilation and vigilant care, as demonstrated in case series of brain death in pregnancy with fetuses brought to term. ${ }^{7}$

Death is determined after cardiac arrest by the loss of the clinical function of heart activity and is based on the absence of circulation. Similar to cardiac arrest, brain death is better understood as brain arrest, based on the complete absence of the clinical functions of the brain. This is documented by the loss of consciousness, unresponsive coma and loss of all brainstem reflexes including the capacity to breathe. It is the maximum clinical expression of irreversible neurological failure and there is no further deterioration of brain function that is possible. While variability in regional ${ }^{1}$ and international ${ }^{2}$ practices is well documented, there is reassuring consistency in the basic clinical criteria. The most reliable imaging correlate of the brain arrest is the absence of cerebral blood flow, and this is a more reliable confirmatory test than the electroencephalogram ${ }^{8}$ and is increasingly recommended in children and adults. ${ }^{9}$

Once brain arrest occurs in the absence of reversible or confounding conditions, the death of that individual is subsequently determined by neurological criteria. This neurological determination of death is the process and procedure to determine death. ${ }^{9}$ Once diagnosed, that individual is medically and legally dead, the option of organ donation should be offered to families and support technology is stopped. It should never be confused with other forms of severe brain injury, such as persistent vegetative state, cortical death or anencephaly. Brain injury in these conditions may be catastrophic and irreversible, but it is not complete as clinical signs of residual brainstem function persist.

Continuing advances in scientific technology force our communities to reflect on the meaning and definition of death. Paradoxically, in many countries with non-heart beating organ donation programs, brain death is now less controversial than cardiac death. The concept of irreversibility and diagnostic criteria are currently being questioned in regard to traditional cardiac arrest and circulatory definitions of death. 10,11 The concept of brain death remains visionary and more valid today than in its origin. The Brazilian PICU experience, while concerning, is reflection of current practices and should be seen as an opportunity for improvement. It is a call for professional education to implement existing national standards.

\section{References}

1. Lago PM, Piva J, Garcia PC, Troster E, Bousso A, Sarno MO, et al. Brain death: medical management in seven Brazilian pediatric intensive care units. J Pediatr (Rio J). 2007;83:133-40.

2. Wijdicks EF. Brain death worldwide: accepted fact but no global consensus in diagnostic criteria. Neurology. 2002;58:20-5.

3. Hornby K, Shemie SD, Teitelbaum J, Doig C. Variability in hospital-based brain death guidelines in Canada. Can J Anaesth. 2006;53:613-9.

4. Taylor RM. Reexamining the definition and criteria of death. Semin Neurol. 1997;17:265-70.

5. Joffe AR, Anton N. Brain death: understanding of the conceptual basis by pediatric intensivists in Canada. Arch Pediatr Adolesc Med. 2006;160:747-52. 
6. Lagiewska B, Pacholczyk M, Szostek M, Walaszewski J, Rowinski W. Hemodynamic and metabolic disturbances observed in brain dead organ donors. Transplant Proc. 1996;28:165-6.

7. Powner DJ, Bernstein IM. Extended somatic support for pregnant women after brain death. Crit Care Med. 2003;31:1241-9.

8. Young GB, Shemie SD, Doig CJ, Teitelbaum J. Brief review: the role of ancillary tests in the neurological determination of death. Can J Anaesth. 2006;53:620-7.
9. Shemie SD, Doig C, Dickens B, Byrne P, Wheelock B, Rocker G, et al. Severe brain injury to neurological determination of death: Canadian forum recommendations. CMAJ. 2006;174:S1-13.

10. DeVita MA. The death watch: certifying death using cardiac criteria. Prog Transplant. 2001;11:58-66.

11. Bernat JL. Are organ donors after cardiac death really dead? J Clin Ethics. 2006;17:122-32. 